\title{
COMPARATIVE DESIGN OF REGULAR STRUCTURED MODIFIED BOOTH MULTIPLIER
}

\author{
Ram RackshaTripathi and S.G.Prakash \\ Department of Electronics \& communication, University of Allahabad-211002
}

\begin{abstract}
Multiplication is a crucial function and plays a vital role for practically any DSP system. Several DSP algorithms require different types of multiplications, specifically modified booth multiplication algorithm. In this paper, a simple approach is proposed for generating last partial product row for reducing extra sign (negative bit) bit to achieve more regular structure. As compared to the conventional multipliers these proposed modified Booth's multipliers can achieve improved reduction in area 5.9\%, power $3.2 \%$, and delay $0.5 \%$ for $8 \times 8$ multipliers. We can also observe that achievable improvement for $16 \times 16$ multiplier in area, power, delay are 4.0\%, 2.3\%, 0.3\% respectively. These multipliers are implemented using verilog HDL and synthesized by using synopsis design compiler with an Artisan TSMC 90nm Technology
\end{abstract}

\section{KEYWORDS}

Digital Multiplier, Modified booth Multiplier (MBE), Power and Delay

\section{INTRODUCTION}

Expansive digital signal processing (DSP) capabilities are a major feature of a large number of System-on-Chip (SoC) designs mainly organize in embedded systems. DSP applications in SoCs range from audio or video processing to wireless communication or adaptive control systems and often make up a considerable part of the system's hardware resources and power consumption. Consequently, various different architecture concepts for their implementation exist, ranging from application-specific components to embedded reconFigureurable [1] hardware or digital signal processors. Each solution represents a specific trade-off between chip area, power consumption, performance, and design effort, currently the most important parameters in SoC design. Starting from our experience with processor data path extensions for wireless sensor network nodes, in this work we investigate the opportunities of reconFigureurable DSP components, which can be reused for different DSP tasks in a SoC. To enhance the processing performance and reducing the power dissipation of systems, designing of multipliers have most challenging task in multimedia and digital signal processing (DSP) applications. Multiplication and multiplication-accumulation (MAC) are very common mathematical operations in many digital signal processing (DSP) applications. For designing of parallel type multipliers there are three common steps to follow: the first one is generating the partial products. Second one is reducing the number of partial product rows. For example, Wallace tree [5]-[6] or Dadda tree. And the third one is adding the remaining two rows of partial products by using a carry propagate adder(e.g., carry lookahead adder) to obtain the final product. 
International Journal of VLSI design \& Communication Systems (VLSICS) Vol.7, No.2, April 2016

The conventional MBE algorithm generates $n / 2+1$ partial product rows rather than $n / 2$ due to the extra partial product bit at the least significant bit position of each partial product row for negative encoding, leading to an irregular partial product array and a complex reduction tree.

Booth encoding is a technique that leads to smaller, faster multiplication circuits, by recoding the numbers that are multiplied. It is the standard technique used in chip design, and provides significant improvements over the "long multiplication" technique. The widely used Booth algorithm is the radix-4 based modified Booth algorithm proposed by McSorley where it reduces the partial products into half. As the number of partial products reduces the number of CSAs required for the compression module, the height of the Wallace tree is also reduced.

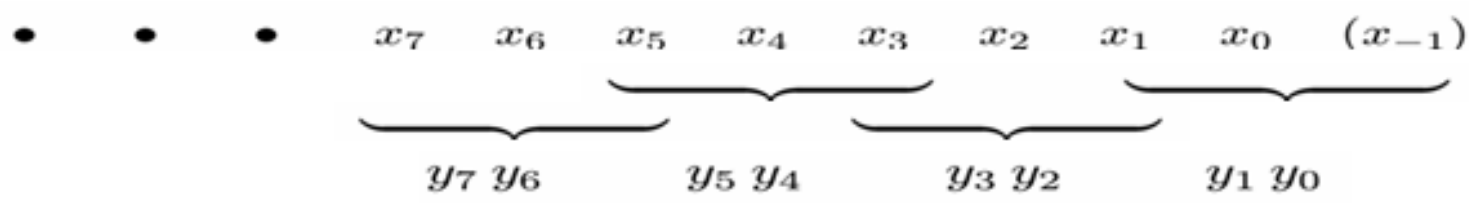

Figure 1. Modified Booth recoding pattern

In Figure.1.Modified Booth algorithm's basic idea is that the bits $Y i$ and $Y_{i-1}$ are recoded into $Z_{i}$ and $Z_{i-1}$, while' $Y_{i-2}$ serves as reference bit. In a separate step, $Y_{i-2}$ and $Y_{i-3}$ recoded into $Z_{i-2}$ and $Z_{i-3}$ with, $Y_{i-4}$ serving as reference bit. This signifies that the modified Booth's encoding partitions input $Y$ into a group of 3-bits with 1-bit overlap and generates the following five signed digits, 2, $1,0,-1$ and -2 . Encoding on the each group reduces the number of partial products by factor of 2 .

Operations on the encoded digits performed with multiplier input $X$ is illustrated in Table 1.

However, it is important to note that there are two unavoidable consequences of using MBE: sign extension prevention and negative encoding. The combination of these two unavoidable consequences results in the formation of one additional partial product row. So that, accumulation of the extra partial product row requires more hardware, and time.

Table 1 : Partial Product Selections and Operations

\begin{tabular}{|c|c|c|}
\hline Recoded digit & Booth's operation on $X$ & $Y 2 i-1 \quad Y 2 i \quad Y 2 i+1$ \\
\hline 0 & Add 0 to $P P$ & $\{000,111\}$ \\
\hline $\begin{array}{l}+1 \\
+2\end{array}$ & $\begin{array}{c}\text { Add X to PP } \\
\text { Shift X left \& add to PP }\end{array}$ & 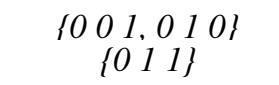 \\
\hline-1 & Add 2's complementary $X$ to $P P$ & 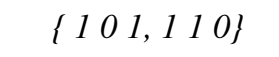 \\
\hline-2 & 2's complementary $X \&$ shift-add & $\left\{\begin{array}{lll}1 & 0 & 0\end{array}\right\}$ \\
\hline
\end{tabular}

To overcome the above consequences, the authors in [2] added the least significant bit of each partial product row with the neg bit of corresponding row to obtained a new least significant bit $\gamma_{i 0}$ and a carry $c_{i}$. Note that both $\gamma_{i 0}$ and $c_{i}$ are generated no later than other partial product bits. Figure 2 depicts the $8 \times 8 \mathrm{MBE}$ partial product array generated by the approach proposed in [2].

Since $c_{i}$ is at the left one bit position of $n e g_{i}$, the required additions in the reduction tree reduced. However, the approach does not remove the additional partial product row. 
International Journal of VLSI design \& Communication Systems (VLSICS) Vol.7, No.2, April 2016

\begin{tabular}{llllllllllllllll}
15 & 14 & 13 & 12 & 11 & 10 & $\mathbf{9}$ & $\mathbf{8}$ & $\mathbf{7}$ & $\mathbf{6}$ & $\mathbf{5}$ & $\mathbf{4}$ & $\mathbf{3}$ & $\mathbf{2}$ & $\mathbf{1}$ & $\mathbf{0}$ \\
\hline & & & & & $\mathrm{A}_{9}$ & $\mathrm{~A}_{8}$ & $\mathrm{~A}_{8}$ & $\mathbf{A}_{7}$ & $\mathbf{A}_{6}$ & $\mathbf{A}_{5}$ & $\mathbf{A}_{4}$ & $\mathbf{A}_{3}$ & $\mathbf{A}_{2}$ & $\mathbf{A}_{1}$ & $\mathbf{T}_{00}$ \\
& & & & $\mathbf{1}$ & $\mathbf{B}_{8}$ & $\mathbf{B}_{7}$ & $\mathbf{B}_{6}$ & $\mathbf{B}_{5}$ & $\mathbf{B}_{4}$ & $\mathbf{B}_{3}$ & $\mathbf{B}_{2}$ & $\mathbf{B}_{1}$ & $\mathbf{T}_{10}$ & $\mathbf{K}_{0}$ & \\
& & $\mathbf{1}$ & $\mathrm{C}_{8}$ & $\mathbf{C}_{7}$ & $\mathbf{C}_{6}$ & $\mathbf{C}_{5}$ & $\mathbf{C}_{4}$ & $\mathbf{C}_{3}$ & $\mathbf{C}_{2}$ & $\mathbf{C}_{1}$ & $\mathbf{T}_{20}$ & $\mathbf{K}_{1}$ & & & \\
& 1 & $\mathbf{P}_{8}$ & $\mathbf{P}_{7}$ & $\mathbf{P}_{6}$ & $\mathbf{P}_{5}$ & $\mathbf{P}_{4}$ & $\mathbf{P}_{3}$ & $\mathbf{P}_{2}$ & $\mathbf{P}_{1}$ & $\mathbf{T}_{30}$ & $\mathbf{K}_{2}$ & & & & \\
& & & & & & & & & $\mathbf{K}_{3}$ & & & & & &
\end{tabular}

Figure 2. Proposed MBE partial product array in [2] for $8 \times 8$ multiplication

The conventional method (complement a binary number and add 1 to the complemented number) will not work properly because the propagation delay of the carry linearly increases with the word size and it would be much greater than the delay to generate the partial products. Therefore, he proposed an extension of the well-known algorithm where all the bits after the rightmost " 1 " in the word are complemented but all the other bits are unchanged. The two's complement of a binary number $001010_{2}$ is $110110_{2}$. For this number, the rightmost " 1 " happens in bit position 1 . Therefore, values in bit positions 2 to 5 can simply be complemented, while values in bit positions 0 and 1 are kept unchanged. Therefore, two's complementation now comes down to finding the conversion signals that are used for selectively complementing some of the input bit.

If the conversion signal at any position is " 0 ", then the value is kept unchanged and, if the conversion signal is " 1 ", then the value is complemented. The conversion signals after the rightmost " 1 " are always 1 . They are 0 otherwise. Once a lower order bit has been found to be a "1," the conversion signals for the higher order bits to the left of that bit position should all be "1."

However, this searching for the rightmost "1" could be as time consuming as rippling a carry through to the MSB since the previous bits information must be transferred to the MSB Therefore, they find a method to expedite this detection of the rightmost " $1 . "$

As we will see, this search for the rightmost "1" can be achieved in logarithmic time using a binary search tree-like structure. They first find the conversion signals for a 2-bit group by grouping two consecutive bits (the grouping always starts from the LSB) from the input and finding the conversion signals in each group, as shown in Figure.3. Then, they find the conversion signals for a 4-bit group (formed by two consecutive 2-bit groups). Then, they find the conversion signals for a 8-bit group (formed by two consecutive 4-bit groups). This divide-and-conquer approach is pursued until the whole input has been covered.

\begin{tabular}{|c|c|c|c|c|c|c|c|c|c|c|c|c|c|c|c|}
\hline & & & & & & & $\times$ & $\begin{array}{l}\mathrm{X}_{7} \\
\mathrm{Y}_{7}\end{array}$ & $\begin{array}{l}\mathrm{X}_{6} \\
\mathrm{Y}_{6}\end{array}$ & $\begin{array}{l}\mathrm{X}_{5} \\
\mathrm{Y}_{5}\end{array}$ & $\begin{array}{l}\mathrm{X}_{4} \\
\mathrm{Y}_{4}\end{array}$ & $\begin{array}{l}\mathrm{X}_{3} \\
\mathrm{Y}_{3}\end{array}$ & $\begin{array}{l}\mathrm{X}_{2} \\
\mathrm{Y}_{2}\end{array}$ & $\begin{array}{l}\mathrm{X}_{1} \\
\mathrm{Y}_{1}\end{array}$ & $\begin{array}{l}\mathrm{X}_{0} \\
\mathrm{Y}_{0}\end{array}$ \\
\hline & & & & & $\overline{\overline{P P_{80}}}$ & $\mathrm{PP}_{80}$ & $\mathrm{PP}_{80}$ & $\mathrm{PP}_{70}$ & $\mathrm{PP}_{60}$ & $\mathrm{PP}_{50}$ & $\mathrm{PP}_{40}$ & $\mathrm{PP}_{30}$ & $\mathrm{PP}_{20}$ & $\mathrm{PP}_{10}$ & $\mathrm{PP}_{00}$ \\
\hline & 1 & 1 & $\overline{P P_{82}}$ & $\begin{array}{l}1 \\
\mathrm{PP}_{72}\end{array}$ & $\begin{array}{l}\overline{P P_{81}} \\
\mathrm{PP}_{62}\end{array}$ & $\begin{array}{l}\mathrm{PP}_{71} \\
\mathrm{PP}_{52}\end{array}$ & $\begin{array}{l}\mathrm{PP}_{61} \\
\mathrm{PP}_{42}\end{array}$ & $\begin{array}{l}\mathrm{PP}_{51} \\
\mathrm{PP}_{32}\end{array}$ & $\begin{array}{l}\mathrm{PP}_{41} \\
\mathrm{PP}_{22}\end{array}$ & $\begin{array}{l}\mathrm{PP}_{31} \\
\mathrm{PP}_{12}\end{array}$ & $\begin{array}{l}\mathrm{PP}_{21} \\
\mathrm{PP}_{02}\end{array}$ & $\mathrm{PP}_{11}$ & $\begin{array}{l}\mathrm{PP}_{01} \\
\text { neg }_{1}\end{array}$ & & nego \\
\hline$\overline{s_{9}}$ & $\mathrm{~S}_{8}$ & $\mathrm{~S}_{7}$ & $\mathrm{~S}_{6}$ & $\mathrm{~S}_{5}$ & $\mathrm{~S}_{4}$ & $\mathrm{~S}_{3}$ & $\mathrm{~S}_{2}$ & $\mathrm{~S}_{1}$ & $S_{0}$ & & neg $_{2}$ & & & & \\
\hline
\end{tabular}

Figure 3 Proposed partial products after removing the last neg. 
However, the approach must additionally develop and design the 2's complement logic. It possibly enlarges the area and delay of the partial product generator and lessens the benefit contributed by removing the extra row.

To remove the extra partial product row the authors in [1] extends the methods proposed in [2] and [3]. To remove the extra partial product row $\mathrm{pp}_{\mathrm{n} / 2}$ due to $\mathrm{c}_{3}$ in Figure 2, they combine the $\mathrm{c}_{i}$ for $i=\mathrm{n} / 2-1$ with the partial product bit $p_{i 1}$ to produce a new partial product bit $\tau_{i 1}$ and a new carry $d_{i}$ can be incorporated into the sign extension bits of $\mathrm{pp}_{0}$. The logic expressions of $\tau_{i 1}$ and $d_{i}$ can be written as

$$
\begin{gathered}
\tau_{i 1}=\text { one }_{i} \cdot \in+t w o_{i} \cdot x_{0}=\overline{\left(\overline{\text { one }}_{i}+\epsilon\right) \cdot\left(\overline{t w o}_{i}+\bar{x}_{0}\right)} \\
d_{i}=\left(\overline{\overline{b_{2 i+1}}}+a_{0}\right) \cdot\left[\overline{\left(b_{2 i-1}+a_{1}\right) \cdot\left(b_{2 i}+a_{1}\right) \cdot\left(b_{2 i}+b_{2 i-1}\right)}\right]
\end{gathered}
$$

And the carry bit is incorporating into the sign extension bits of first partial product row. The maximal value of these sign extension bits are 100 so the addition of carry bit to sign extension bits will never produce an overflow.

\section{PROPOSED WORK}

For recording of MBE, We need at least three signal to represent the digit set $\{-2 X,-1 X, 0,1 X$, $2 \mathrm{X}\}$. Many different ways have been developed. But the proposed selector circuit in[15] is not efficient to design the multiplier because it can not satisfy all the encoding conditions. For that purpose we redesign the selector circuit. The Booth encoder and selector circuits for the implementation of proposed MBE multiplier are shown in Figure. 4, Figure. 5 respectively.

To have a more regular least significant part of each partial product row , the authors in [2] added the least significant bit ${ }_{i 0}$ with neg $_{\mathrm{i}}$ in advance and obtained a new least significant bit $\gamma_{i 0}$ and a carry $c_{i}$. However, the approach does not remove the additional partial product row. i.e., $\mathrm{n} / 2+1$ row still present. To remove this extra row, we are using the advantages of [3]. For generating a 2 's complement of a number generally we have two methods. The first method is converting each bit of a binary number and add ' 1 ' to the least significant bit. And the second method is that search for a right most binary ' 1 ' and all the bits after right most ' 1 ' in the word are complemented but all the other bits are unchanged.

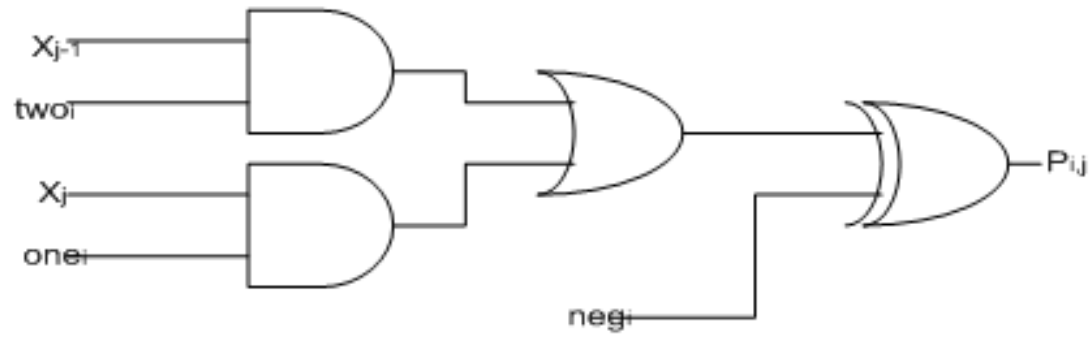

Figure 4. Proposed MBE selector circuit 
International Journal of VLSI design \& Communication Systems (VLSICS) Vol.7, No.2, April 2016
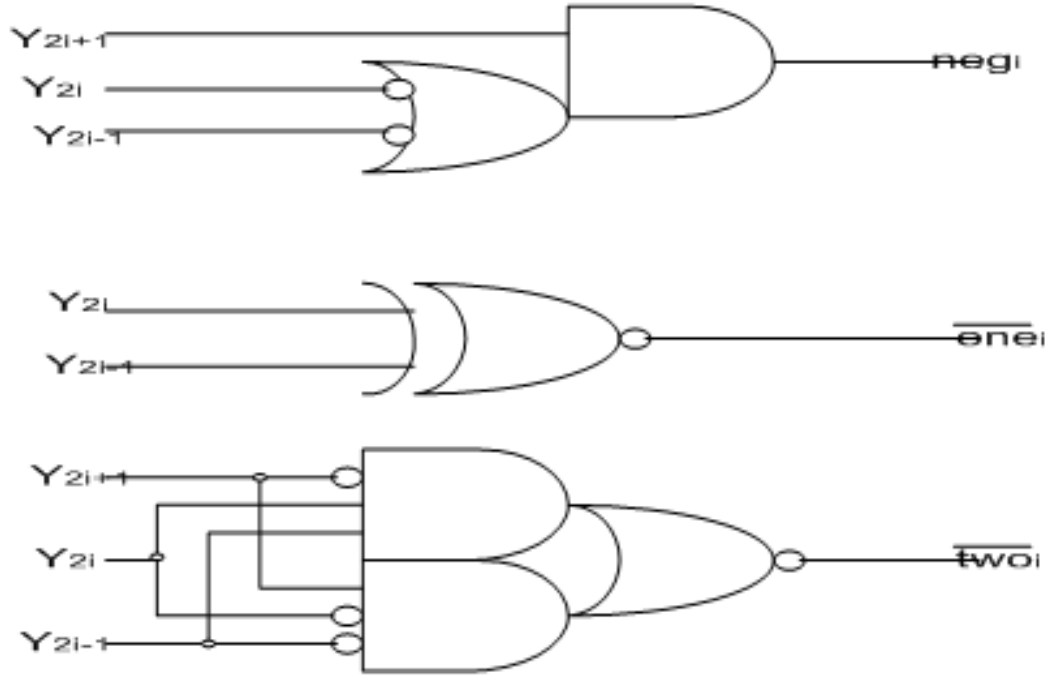

Figure 5. MBE encoder proposed in [15]

In this brief, for our proposed MBE multiplier, we combine the two methods for generating the last partial product row. For the generation of the first two least significant bits of last partial product row, we are using second method i.e. , searching for right most ' 1 ' in the two least significant bits and replace the bits according the Table 2 . The corresponding circuits to generate $p_{i 1}, \bar{s}$ for the proposed multiplier are depicted in Figure 6, Figure 7.

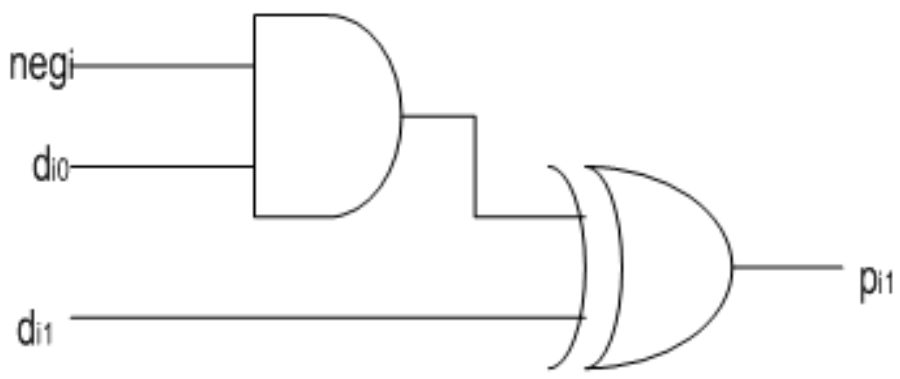

Figure 6. Proposed circuit to generate $\boldsymbol{p}_{\mathrm{i} 1}$

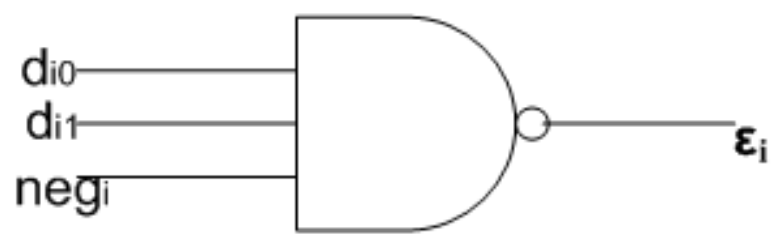

Figure 7. Proposed circuit to generate $\varepsilon_{\mathrm{i}}$ 
International Journal of VLSI design \& Communication Systems (VLSICS) Vol.7, No.2, April 2016

Table. 2. Proposed MBE multiplier truth table for generation of partial product bits pil pio

\begin{tabular}{lll|lll}
\hline $\boldsymbol{n e g}_{\boldsymbol{i}}$ & $\boldsymbol{d}_{\boldsymbol{i l}}$ & $\boldsymbol{d}_{\boldsymbol{i} \boldsymbol{0}}$ & $\boldsymbol{P}_{\boldsymbol{i l}}$ & $\boldsymbol{P}_{\boldsymbol{i} \boldsymbol{0}}$ & $\boldsymbol{\varepsilon}_{\boldsymbol{i}}$ \\
\hline 0 & 0 & 0 & 0 & 0 & 0 \\
0 & 0 & 1 & 0 & 1 & 0 \\
0 & 1 & 0 & 1 & 0 & 0 \\
0 & 1 & 1 & 1 & 1 & 0 \\
1 & 0 & 0 & 0 & 0 & 0 \\
1 & 0 & 1 & 1 & 1 & 0 \\
1 & 1 & 0 & 1 & 0 & 0 \\
1 & 1 & 1 & 0 & 1 & 1 \\
\hline
\end{tabular}

Where $\varepsilon_{\mathrm{i}}$ is the carry bit and $i=\mathrm{n} / 2-1$. If any carry is generated, since the weight of $\varepsilon_{\mathrm{i}}$ is $2^{\mathrm{n},}$ which is equal to the weight of sign extension bit $s_{0}$ at bit position $n, \varepsilon_{\mathrm{i}}$ can be incorporated with the sign extension bits of first partial product row. So that the carry bit do not propagate up to the $2 \mathrm{n}^{\text {th }}$ bit position. Since the maximal value of sign extension bits $\bar{s}_{0} \mathrm{~s}_{0} \mathrm{~s}_{0}$ in the first partial product row is 100 so that the addition of these bits with $\varepsilon_{\mathrm{i}}$ will never produce an overflow. The total conversion of sign extension bits proposed in [1] is shown in Table 3 and corresponding circuit is given in Figure 8.

Table 3.Truth table for new sign extension bits

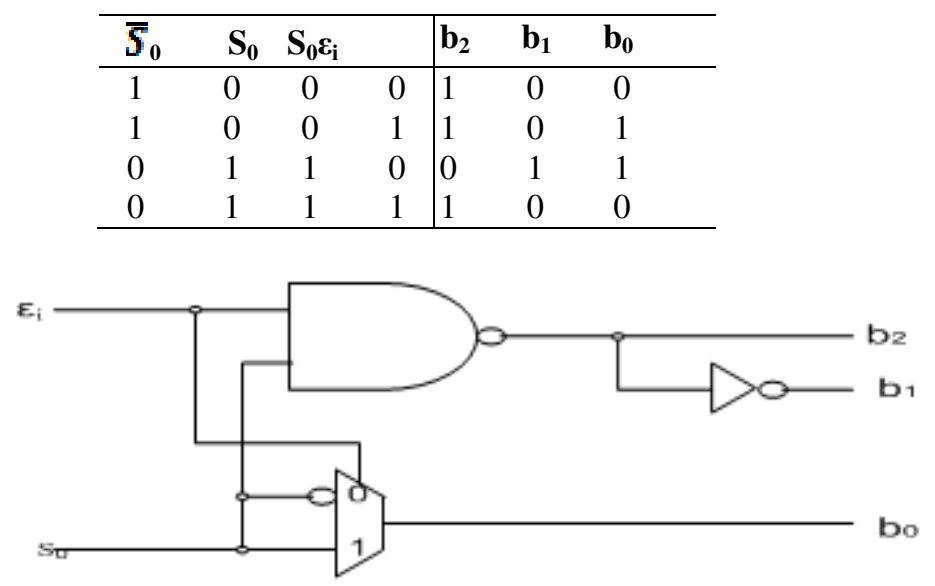

Figure 8. Circuit to generate $b_{2} b_{1} b_{0}$ proposed in [1]

\section{SIMULATION RESULTS AND DISCUSSION}

The simulation results for different numbers of bits for proposed modified Booth's multipliers for regular partial product array is shown in Figure. 9, 10, 11 and 12 below. Simulation results for 8bit proposed multipliers for generating regular partial product array.

The partial products of multipliers are generally generated by using two-input AND gates or a MBE algorithm [7]-[9]. The latter has widely been adopted in parallel multipliers since it can reduce the number of partial product rows to be added by half, thus reducing the size and enhancing the speed of the reduction tree. However, the conventional MBE algorithm generates $n / 2+1$ partial product rows rather than $n / 2$ due to the extra partial product bit (negative bit) at the 
International Journal of VLSI design \& Communication Systems (VLSICS) Vol.7, No.2, April 2016

least significant bit position of each partial product row for negative encoding, leading to an irregular partial product array and a complex reduction tree. Some approaches [1]-[3] have been proposed to generate more regular partial product arrays, for the MBE multipliers. The authors in [3] added the least significant bit of each partial product row with negative bit in advance, but the method does not remove the additional partial product row. In the proposed work in [2] the authors proposed a method to directly generates the two's complements of a negative row, but it requires extra hardware. In [1] author almost overcome the above problems by extending the methods proposed in [2] and [3]. The carry bit generated at the last partial product row in [3] is incorporating into the sign extension bits of first partial product row. But the proposed circuits were not satisfying all the conditions. So we redesigned the circuits by a simple method for reduction of last negative bit.

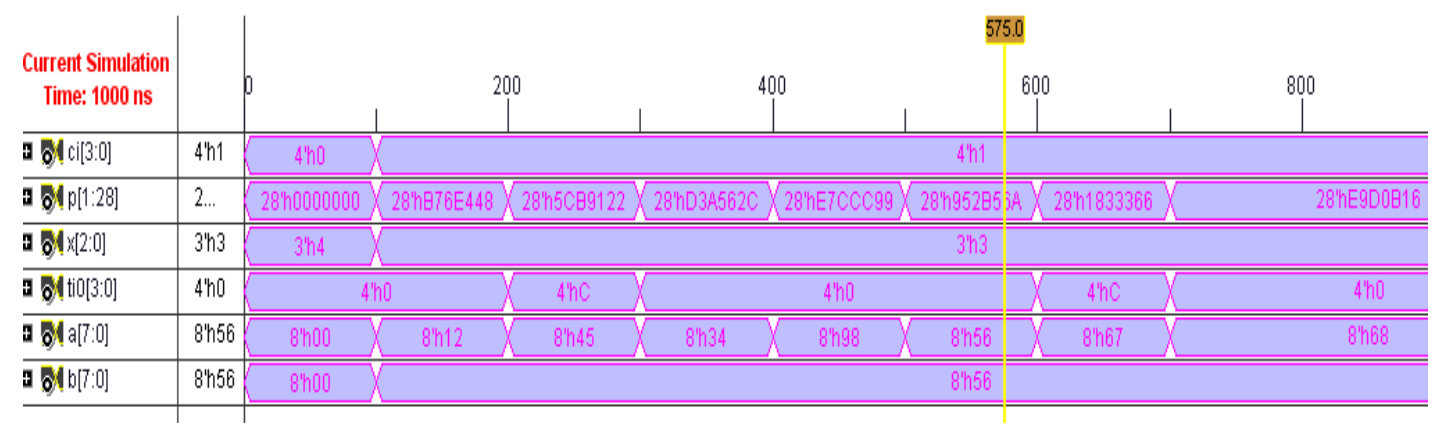

Figure 9. Simultion results for proposed 8 x 8 multiplier PPA generation

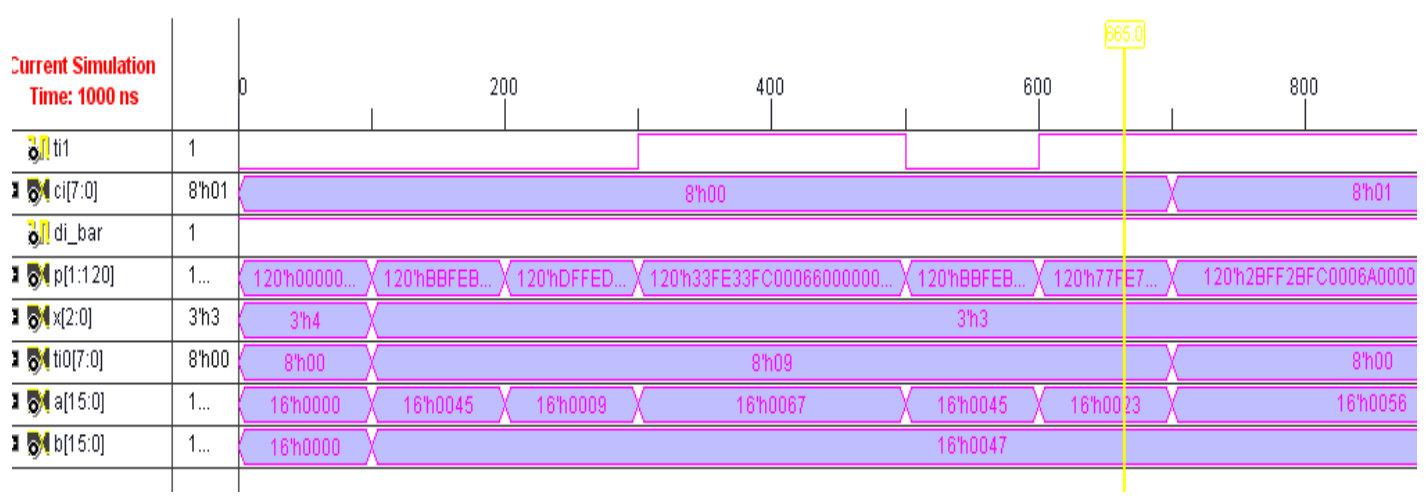

Figure10. Simultion results for proposed 16 x 16 multiplier PPA generation

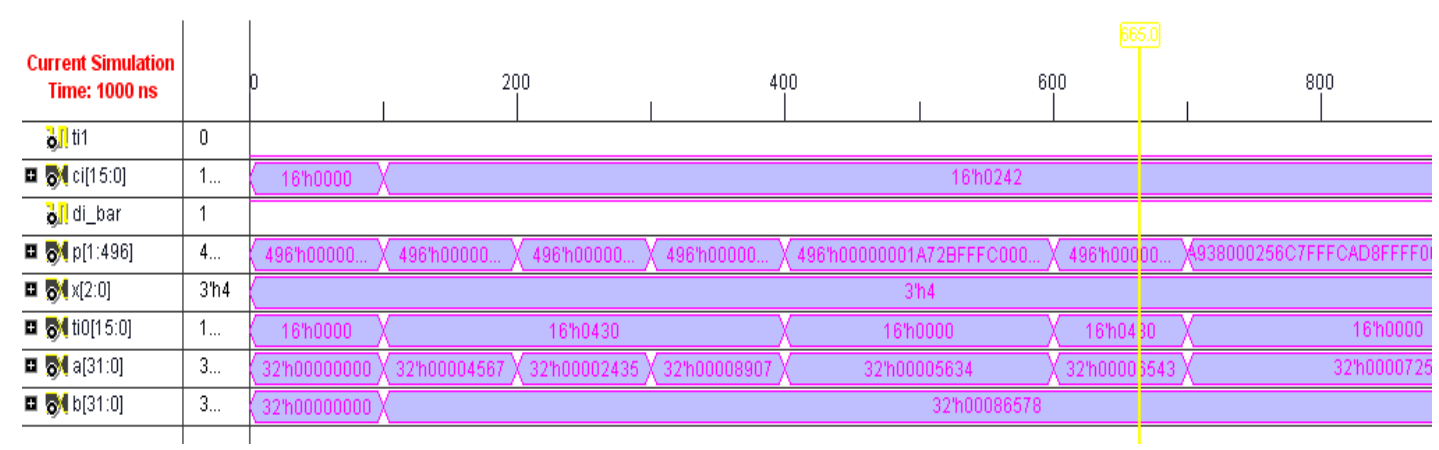

Figure 11. Simultion results for proposed 32 x 32 multiplier PPA generation 
International Journal of VLSI design \& Communication Systems (VLSICS) Vol.7, No.2, April 2016

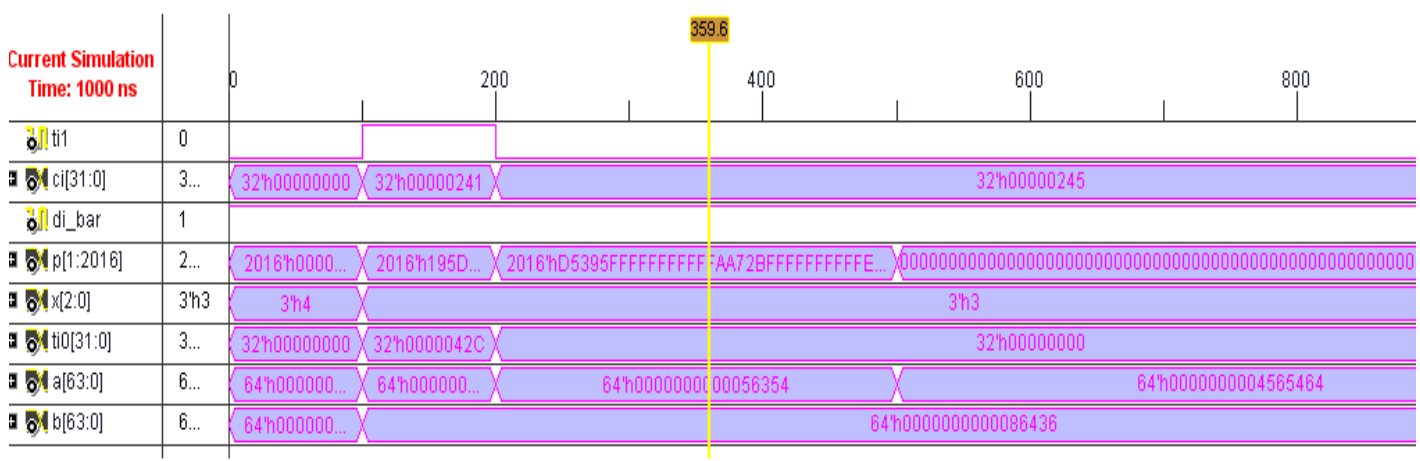

Figure 12. Simultion results for proposed 64 x 64 multiplier PPA generation

For comparison, we have implemented the multiplier proposed in [1]. For the implementation of the other partial product except for a few of partial product bits that are generated by different schemes to regularize the partial product array, the other partial product bits are generated using the proposed MBE selector circuits for all the multipliers. These multipliers are modelled in verilog HDL and synthesized by using synopsys design compiler with Artisan TSMC 90nm technology.

Table 4 : Experimental Results of Generation of Partial Product Bits

\begin{tabular}{lllll}
\hline & $\begin{array}{l}\text { Input } \\
(\mathbf{n}-b i t)\end{array}$ & $\begin{array}{l}\text { Area } \\
\left(\boldsymbol{\mu m}^{2}\right)\end{array}$ & $\begin{array}{l}\text { Power } \\
(\mathbf{m w})\end{array}$ & $\begin{array}{l}\text { Delay } \\
(\mathbf{n s})\end{array}$ \\
\hline Ref_1 & 8 & 1046 & 0.306 & 0.68 \\
& 16 & 3550 & 1.176 & 0.72 \\
& 32 & 14029 & 4.328 & 0.86 \\
& 64 & 60918 & 19.007 & 5.62 \\
\hline Proposed & 8 & 947 & 0.295 & 0.64 \\
& 16 & 3259 & 1.0796 & 0.71 \\
& 32 & 3988 & 4.254 & 0.84 \\
& 64 & 58275 & 19.007 & 5.61 \\
\hline
\end{tabular}

The implementation results in Table 5 demonstrated that the improved reduction in area 5.9\%, power $3.2 \%$, and the delay is $0.5 \%$ as compared to proposed multiplier in [1] for $8 \times 8$ multiplier. We can also observe that achievable improvement for $16 \times 16$ multipliers in area, power, and delay are $4.0 \%, 2.3 \%$, and $0.3 \%$ respectively. For the comparison purpose for the generation of partial products for higher order bits we implemented up to $64 \times 64$ bit multiplier. As shown in Table 4. It shows that for higher order multipliers the percentage improvement with respect to the area, delay, and power is respectable.

Table 5 : Synthesis Report of Proposed Multiplier

\begin{tabular}{lllll}
\hline & $\begin{array}{l}\text { Input } \\
(\mathbf{n}-\mathbf{b i t})\end{array}$ & $\begin{array}{l}\text { Area } \\
\left(\boldsymbol{\mu m}^{\mathbf{2}}\right)\end{array}$ & $\begin{array}{l}\text { Power } \\
(\mathbf{m w})\end{array}$ & $\begin{array}{l}\text { Delay } \\
(\mathbf{n s})\end{array}$ \\
\hline Ref_1 & 8 & 2868 & 1.186 & 3.80 \\
& 16 & 10264 & 5.923 & 5.34 \\
\hline Proposed & 8 & 2696 & 1.148 & 3.78 \\
& 16 & 10325 & 5.785 & 5.32 \\
\hline
\end{tabular}


International Journal of VLSI design \& Communication Systems (VLSICS) Vol.7, No.2, April 2016

\section{CONCLUSION}

Multiplication is a frequently encountered operation, especially in signal processing applications. So the development of a multiplier is vital for applications in portable mobile devices such as personal multimedia players, cellular phones, digital cam coders and digital cameras. Many designs have been proposed for Booth encoder and selector logic using CMOS over the past decades. But those designs when implemented in CMOS resulted in higher transistor count. In our research, Booth encoder and selector logic occupies one third of the entire multiplier architecture. So careful optimization of these logic parts will result in a considerable reduction of hardware.

We proposed new circuits for removing the extra partial product array because of negative compensation bit for negative coding. The results obtained here are compared to proposed results in [1]. It shows that proposed multipliers are well regularly structured and complexity of the design is reduced. Also observed that the reduction in area, power, delay are 5.9\%, 3.2\%, 0.5\% respectively for $8 \times 8$ multipliers and it is $4.0 \%, 2.3 \%, 0.3 \%$ for $16 \times 16$ bit multipliers. Apart from above we also presented the new circuit for MBE selector.

\section{REFERENCES}

[1] S. R. Kuang, J. P. Wang, C.Y. Guo, "Modified Booth multipliers with a regular partial product array,” IEEE Trans.CircuitsSyst.II , vol. 56, pp.404 - 408, May 2009.

[2] W. C. Yeh and C.-W. Jen, "High-speed Booth encoded parallel multiplier design," IEEE Trans. Comput., vol. 49, no. 7, pp. 692-701, Jul. 2000.

[3] J.-Y. Kang and J.-L. Gaudiot, "A simple high-speed multiplier design," IEEE Trans. Comput., vol. 55, no. 10, pp. 1253-1258, Oct. 2006.

[4] C. S. Wallace, “A suggestion for parallel multipliers," IEEE Trans. Electron. Comput., vol. EC-13, no. 1, pp. 14-17, Feb. 1964.

[5] O. Hasan and S. Kort, "Automated formal synthesis of Wallace tree multipliers," in Proc. 50th Midwest Symp. Circuits Syst., pp. 293-296, 2007.

[6] J. Fadavi-Ardekani, "M $\times \mathrm{N}$ Booth encoded multiplier generator using optimized Wallace trees," IEEE Trans. Very Large Scale Integr. (VLSI)Syst., vol. 1, no. 2, pp. 120-125, Jun. 1993.

[7] F. Elguibaly, "A fast parallel multiplier-accumulator using the modified Booth algorithm," IEEE Trans. Circuits Syst. II, vol. 47, no. 9, pp. 902-908, Sep. 2000.

[8] K. Choi and M. Song, "Design of a high performance $32 \times 32$-bit multiplier with a novel sign select Booth encoder," in Proc. IEEE Int. Symp.Circuits Syst., vol. 2, pp. 701-704, 2001.

[9] Y. E. Kim, J. O. Yoon, K. J. Cho, J. G. Chung, S. I. Cho, and S. S. Choi, "Efficient design of modified Booth multipliers for predetermined coefficients," in Proc. IEEE Int. Symp. Circuits Syst., pp. 2717-2720, 2006.

[10] O. Salomon, J.-M. Green, and H. Klar, "General algorithms for a simplified addition of 2's complement numbers,” IEEE J. Solid-State Circuits, vol. 30, no. 7, pp. 839-844, Jul. 1995. 
International Journal of VLSI design \& Communication Systems (VLSICS) Vol.7, No.2, April 2016

[11] E. de Angel and E. E. Swartzlander, Jr., "Low power parallel multipliers," in Workshop VLSI Signal Process. IX, pp. 199-208, 1996.

[12] A. A. Farooqui and V. G. Oklobdzija, "General data-path organization of a MAC unit for VLSI implementation of DSP processors," in Proc. IEEEInt. Symp. Circuits Syst., vol. 2, pp. 260-263, 1998.

[13] S.-F. Hsiao, M.-R. Jiang, and J.-S. Yeh, "Design of high-speed low-power 3-2 counter and 4-2 compressor for fast multipliers," Electron. Lett., vol. 34, no. 4, pp. 341-343, Feb. 1998.

[14] C.-H. Chang, J. Gu, and M. Zhang, "Ultra low-voltage low-power CMOS 4-2 and 5-2 compressors for fast arithmetic circuits," IEEE Trans. CircuitsSyst. I, Reg. Papers, vol. 51, no. 10, pp. 1985-1997, Oct. 2004.

[15] Z. Huang and M. D. Ercegovac, "High-performance low-power left-to-right array multiplier design," IEEE Trans. Comput., vol. 54, no. 3, pp. 272-283, Mar. 2005.

[16] CIC Referenced Flow for Cell-based IC Design, 2008, Taiwan: Chip Implementation Center, CIC. Document no. CIC-DSD-RD-08-01.

[17] G.Goto et al., "A4.1ns compact 54 X54-b Multiplier Utilizing Sign-select Booth Encoders," IEEE J. Solid-state Circuits.,vol. 32, no. 11, pp. 1,676-1,682, Nov. 1997.

[18] P. F. STELling, C. U. Martel, V. G. Oklobdzija, and R. Ravi, "Optimal Circuits for parallel Multipliers,” IEEE Trans. Computers., vol. 47, no. 3, pp. 273-285, Mar. 1998.

[19] J. Cavanagh, "Digital Design and Verilog-HDL Fundamentals", CRC Press Taylor and Francis Group 2008.

[20] C. Senthilpari, A. K. Singh, K. Diwakar, "Design of a low power, high Performance, $8 \times 8$ bit multiplier using a Shannon-based adder cell”, Microelectronics Journal, Vol. 39, Issue 5, pp.812-821, May 2008.

[21] William Stallings, "Cryptography and Network Security Principles and Practices", Fourth Edition, Prentice Hall, 2005.

[22] Shu Lin, Daniel J. Costello, "Error Control Coding: Fundamentals and Applications", Prentice Hall, 1983.

[23] Jean-Pierre Deschamps, Gery Jean Antoine Bioul, Gustavo D. Sutter, "Synthesis of Arithmetic Circuits- FPGA, ASIC, and Embedded Systems", A John Wiley \& Sons, Inc., 2006.

[24] www.xilinx.com/

[25] https: //solvnet.synopsys.com 